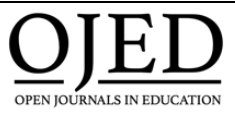

Volume 4, Issue 1 (2020), pp. 10-15

International Journal of

Entrepreneurship and Economic Issues

ISSN: 2616-0048 Print/ ISSN: 2631-231X Online

\title{
ECHO Education: A Multisectoral Effort Ensuring Educational Success During the COVID-19 Pandemic
}

\author{
Edward L. Harris \\ Kathrine A. Curry \\ Jentre J. Olsen \\ Oklahoma State University-Tulsa, USA
}

\begin{abstract}
Unforeseen challenges caused by the COVID-19 pandemic have taken a significant toll on people across the world. This essay deals with a holistic, multisectoral effort that one US higher education institution took to collaborate with national, state, and local entities in order to serve their State's constituents during the pandemic. The goal of this essay is to describe this endeavor as a model that can serve other sectors, agencies and institutions worldwide.
\end{abstract}

Keywords: COVID-19, ECHO, Education, Pandemic, Professional Development, Workforce Development.

The COVID-19 pandemic continues to have an impact on educational systems worldwide and led to massive school closures that disrupted traditional educational practices. In Oklahoma, multiple sectors, including government, health, communication, and education, collaborated to support educational institutions as they adjusted to rapid change. The following 
narrative explains the holistic approach that addressed the multifaceted challenges brought on by this pandemic.

\section{A TRANSFERABLE MODEL TO ADDRESS CONSTITUENT NEEDS IN A PANDEMIC}

The COVID-19 pandemic tested the capacity of educational institutions across the globe in their ability to educate students remotely. Unfortunately, few systems were prepared for such disruptive change, and many educational institutions could not adequately address the needs of teachers, parents, and students (Hobbs \& Hawkins, 2020). The consequences were most devastating in low-income, remote areas typically characterized by inadequate financing, internet inaccessibility, and inequitable professional development opportunities to address these issues.

To respond to the crisis in Oklahoma, the educational leadership faculty at Oklahoma State University (OSU) partnered with medical, educational, and governmental entities to provide sustained professional development, medical knowledge, and educational resources for Oklahoma educators in record setting scope and efficiency. The framework for this anomalous success story actually began many years prior to the pandemic in an adjacent state.

In 2003, Dr. Sanjeev Arora, a renowned hepatologist at the University of New Mexico, was frustrated that he could only serve a fraction of the hepatitis $\mathrm{C}$ patients in his state. Consequently, he invented a system whereby teams of experts could provide guided professional direction and mentoring via Zoom technology to health care workers in remote areas to meet their patients' needs. This free, mentoring model is referred to as the Extension for Community Healthcare Outcomes, or simply, Project ECHO. Currently, 429 Project ECHO bases serve the United States and 33 countries across the globe and address a plethora of mental, emotional, and medical conditions.

In Oklahoma, Project ECHO activities are coordinated through the OSU Center of Health Sciences (OSU-CHS). OSU-CHS began offering ECHO Medical lines in 2016 to serve rural areas in the State. In 2018, a team of OSU educational leadership professors partnered with OSU-CHS to utilize the ECHO model to provide professional development for rural educational leaders. This strand of ECHO Education was branded TeleED (Educational Development), and through Zoom technology, TeleED linked expert specialists, or "hub-team" members, with educational practitioners in local communities referred to as "spoke" sites. 
Staying true to ECHO standards, four principles guide TeleED processes: maximize available technologies to leverage scarce resources, disseminate best practices to democratize knowledge, use case-based learning to master complexity, and develop a web-based database to monitor outcomes and curate digital archives and resource materials.

Before the pandemic, TeleED hosted biweekly, one-hour sessions presented in ECHO's time-honored, three-part format: case presentation, didactic, and discussion/recommendations. Cases focus on anonymous, reallife problems from practice that are context-based and contain multiple factors including technical, economic, social, ethical, and legal. The didactic is a theory-to-practice presentation typically delivered by a hub-team member or guest presenter. Both the case and didactic promote interactive hub/spoke interaction leading to recommendations for best practices and strategies. Application of ECHO principles and processes help cultivate a dynamic, professional learning community in which "all teach; all learn." Moreover, the model bridges digital divides because rural educators can participate in ECHO sessions and network with professional peers statewide though their computers and all mobile devices.

In order to collaboratively expand the influence of ECHO Education efforts across Oklahoma, the TeleED group partnered with ECHO's national education base in Albuquerque, Oklahoma's Governor, the Department of Education, and the School Administrators' Association, as well as local foundations and other state and local entities. Consequently, additional ECHO lines were developed between 2018 and 2020 to meet diverse needs across the State, including TeleSPED for special education and TeleEDGE for general education. When the pandemic disrupted everyday life across the world, these previously established platforms provided the infrastructure necessary for professional development, networking, and adaptability for Oklahoma citizens.

During the pandemic, Oklahoma educational institutions closed their doors. All ECHO Medical and ECHO Education teams, existing partner associations, local businesses and foundations allied to provide vital multisectoral support to Oklahoma constituents. As educators grappled for resources and support to address their many questions and concerns associated with the crisis, participant attendance at each ECHO Education line (TeleED, TeleSPED, and TeleEDGE) skyrocketed from the usual 20 participants per session to sometimes over 500 participants per session. Prior to the pandemic, each of the ECHO lines met bi-weekly. However, as the abundance of new information surged and the pace of understanding surrounding COVID-19 accelerated, all of the lines began meeting on a 
weekly basis. Topics included COVID updates, medical and legal guidance, remote learning planning and delivery, capacity building, student assessments and motivation, community needs, mental health, and engaging families who are assuming more responsibility for their children's learning.

The outcomes and benefits of the combined efforts of ECHO Medical and ECHO Education have been staggering. Oklahoma ECHO Director, Dr. Tara Jackson, and Medical Director, Dr. Joe Johnson, estimated that ECHO mentoring and professional development have resulted in cost savings of over $\$ 75,000,000$ to Oklahoma. Medical benefits included decreased COVID-19 infection rates by providing evidence-based bestpractices for prevention and rapid treatment protocols, dissemination protocols for the Governor's Surge Task Force, and statewide, internetworked service lines for medical and emergency services and medical examiners. ECHO Education lines served and continue to serve every Oklahoma county and have reached over 5,000 participants. These lines have allowed for a consistent channel of clear and timely communication between educational leaders and practitioners, medical doctors, state officials, and experts in mental health, which proved to be invaluable during uncertain times.

\section{IMPLICATIONS}

Networks established through ECHO Education lines enhanced efficacy for remote learning and provided a platform for sharing multifaceted resources. As educators continue to experience the changes imposed by the pandemic and imagine a "post COVID-19" future, implications for practice, research, and theory abound.

Regarding implications for practice, the ECHO model is extremely transferable and can be adapted to multiple sectors and emerging needs (Project ECHO, 2020). In Oklahoma, topics were chosen in response to the voiced needs of educators. Teams of experts, including medical professionals and state officials, were quickly recruited to provide pertinent, up-to-the-minute information. Educational experts provided support for remote learning. School psychologists addressed social emotional and psychological needs of teachers, students, and families.

The need for enhanced school-family engagement was reinforced in the crisis. Families are an important component in facilitating student learning (Gordon \& Louis, 2009), and an opportunity exists to re-imagine family/school relationships. Thus, in fall 2020, OSU will begin a fourth ECHO Education line, TeleNGAGE. TeleNGAGE will connect families and 
schools to promote shared understandings and serve as a platform for schools and families to understand their roles and responsibilities as they adjust to education's "new normal."

Implications for theory and research are also plentiful. Professional Learning Communities (PLCs), Communities of Practice (CoPs), and Adult Learning Theory provide frameworks to move the field forward when understanding professional development through ECHO lines. PLCs are a viable means for continual learning for teachers and principals (Servais, Sanders, \& Derrington, 2009). The "all teach; all learn" ECHO mentality provides a platform for rich unidirectional dialogue that promotes sustainable professional development and relationships over time. The pandemic brought the educational community together, yet the effectiveness of these initial relationships for professional learning over time is still to be determined and merits further research.

Similarly, by design, CoPs are voluntary, self-organizing groups of practitioners who coalesce around a shared goal (Snyder \& Wenger, 2004). As made evident by the COVID-19 pandemic, ECHO Education lines in Oklahoma were teeming with eager participants ready to solve immediate problems. The number of volunteer participants indicates that there is power in collective professional learning, especially when traditional learning platforms are limited. As we move into the future, real-time virtual professional learning will continue to grow, and research on the effectiveness of these platforms and best practices within online PLCs and CoPs is imperative.

In summary, this experience of utilizing ECHO during the recent COVID-19 health crisis reinforced the understanding that the workforce, in all sectors, needs ongoing, up-to-date, and sustainable training. When professional development is flexible enough to respond to the changing needs of its audience, the potential for capacity building expands exponentially. Not only are participants motivated to engage because of the immediate applicability of information and training presented, but also casebased learning is memorable and provides a platform for transferability to a variety of challenges and contexts.

\section{REFERENCES}

Gordon, M. F., \& Louis, K. S. (2009). Linking parent and community involvement with student achievement: Comparing principal and teacher perceptions of stakeholder influence. American Journal of Education, 116(1), 1-31. 
Hobbs, T. D., \& Hawkins, L. (2020, June 10). The results are in for remote learning: It didn't work. Wall Street Journal. https: //www.wsj.com

Project ECHO (2020, June 1). Start an ECHO. https://echo.unm.edu/jointhe-movement/start-an-echo

Servais, K., Sanders, K., \& Derrington, M. L., (2009). Professional learning communities: Concepts in action in a principal preparation program, an elementary school team, a leadership team, and a business partnership. International Journal of Educational Leadership Preparation, 4(2). http:// cnx.org/content/m21925/1.1/

Snyder, W.M., \& Wenger, E. (2004). Our world as a learning system: A communities-of-practice approach. In C. Blackmore (Ed), Social Learning Systems and Communities of Practice, (pp. 107-124). Springer.

EDWARD HARRIS, PhD, is a Professor and Williams Chair of Educational Leadership in the College of Education and Human Sciences, Oklahoma State Universality, USA. His research interests focus on organizational culture and educational innovation. Email: ed.harris@okstate.edu

KATHERINE CURRY, EdD, is an Associate Professor and John A. and Donnie Brock Professorship in Educational Leadership and Policy Studies of Educational Leadership at Oklahoma State University, USA. Her research interests include school/family engagement, social networks, educational leadership and reform, and school/community partnerships. Email: katherine.curry@okstate.edu

JENTRE OLSEN, PhD, is an Assistant Professor and Brock Chair of Innovative Educational Leadership at Oklahoma State University, USA. His research focuses primarily on innovative approaches to school leadership, namely the effects of intentional interaction on principals' support of teacher psychological needs. Email: jentre.olsen@okstate.edu 